\title{
3D Flow Modeling of the First Trifurcation Made in Nepal
}

\author{
RK Malik, Paras Paudel
}

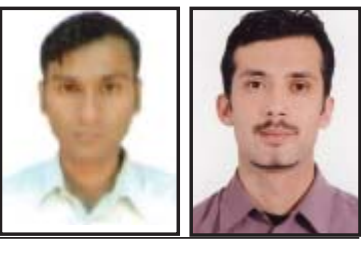

RK Malik

Paras Paudel

\begin{abstract}
The foremost objective of the study was to find out the most efficient profile of trifurcation in given constraints of pressure, velocity and layout of the overall geometry. The study was done for the 3.2 MW Madi Khola Hydropower Project of Gandaki Hydropower Development Co. Pvt. Ltd. The 3 Dimensional Flow modeling of the trifurcation was based on the application of Computational Fluid Dynamics (CFD).
\end{abstract}

The loss in the Trifurcation greatly depends upon its geometrical configuration. The research started with a general profile and the flow pattern generated inside it was studied with the help of 3 Dimensional Flow modeling. The extent of vortex zone formation inside the trifurcation indicates the loss inside trifurcation. The profile of the trifurcation was hence changed to reduce the vortex formation as far as possible, till we get minimum possible loss. The profile under study should meet maximum flow efficiency under the physical constraints of fabrication. The flow efficient profile was then analyzed to capture the stress amplification near junction. The reinforcing element in the form of steel T-section was added of different sectional values till the stress was within allowable limits under severe conditions.

Key words: Symmetrical Trifuraction, trifurcation, Computational fluid dynamics, hydropower, Nepal

\section{Introduction}

Project layout and powerhouse orientation decide what kind of penstock branching would be most suitable for the highest safety and minimum head loss. Usually in case of branching for 3 or more units, a number of unsymmetrical bifurcations are used one after another along the penstock alignment. It involves relatively less analytical works during design and is easy to fabricate. But project layout can dictate the other way around, as in case of 3.2 MW Madi Khola Hydropower Project in Kaski district, Nepal, which is already nearing the completion of construction. In this project orientation of the powerhouse with respect to the penstock alignment is such that only a symmetrical trifurcation can be used to feed water to three equal capacity Pelton turbines. Madi trifurcation has become the first trifurcation designed and fabricated within Nepal. It was manufactured and installed by Radha Structures and Engineering Works Pvt. Ltd.

Design of an element of water conveyance system constitute of hydraulic and structural analyses. Hydraulic analysis for penstock branching (bifurcation and trifurcation) is considered unimportant and is found mostly avoided in small hydropower projects of Nepal. But the fact is that hydraulic analysis here is as important as structural analysis. Structural analysis optimizes the initial cost through right selection of steel thicknesses, whereas hydraulic analysis minimizes the head loss through selection of best possible geometry. Head loss in the branching entails a constant loss of money for as long as the plant runs, and this loss, in long run, is many times higher than the cost of the structure itself. Large hydropower projects do not only conduct hydraulic analysis but also go for model tests. But small project of capacities 1-10 MW are recommended at least to conduct hydraulic analyses for the critical components of water conveyance system. Bifurcation and trifurcation are among such components. They are used near the powerhouse under high pressure head added by pressure surge due to water hammer. Besides, in case of Pelton turbines, there are free water jets downstream to the manifold, which convert almost all of the available head into velocity head. Under this condition the water exerts force on manifolds due to change in momentum of water and can be evaluated from principle of conservation of momentum. This net force must be resisted by the manifold system and the concrete block holding manifold.

The structural analysis of the manifolds is necessary but not sufficient if we consider the long term operational benefits in terms of power outcome and the performance of the plant. The vibration problem caused due to unnecessary eddies developed can cause huge losses of pressure head downstream of the manifold. The profile of the manifolds affects the loss in the available water head significantly. This loss can decrease the potential plant capacity. The profile selection process can be done either by experimental analysis on reduced scale manifolds model test at lab or by numerical modeling of the fluid flow. The former option is rather expensive and may not be feasible every time. It is preferable to select best profile by tuning it with CFD solver and then follow reduced scale model test for the confirmation of flow parameters.

At the time of valve closure the velocity reduces to zero within time interval of valve closure. This phenomenon now converts velocity head to pressure head again. The 
pressure magnifies due to abrupt change in the velocity. The magnified pressure moves to up stream with certain velocity which depends up on bulk modulus of water and its density. The trifurcation has to resist this magnified pressure. The hoop stresses and longitudinal stress and the combined stress due to increased pressure must be below the tolerable limit as specified in Steel Structure Design Codes. At the zone of the junction of bifurcation the simple analysis approach can not catch the local stress concentration and there are chances of underestimation of such valuable stress concentration. Finite element method can be used to capture Stress Concentration near junction of trifurcation using finer mess near junction.

\section{Design \& Analysis Criteria}

The pressure losses at junction in manifolds are not analyzed and neglected which is the normal practice in hydropower of Nepal. These losses are significant in obtaining high plant capacity. Before starting design and analysis of the trifurcation, the constraining parameter, such as space available and the position of turbine from the existing site condition, are used to select the trifurcation layout. The normal practiced profile is first used in detailed numerical analysis to understand how the profile affects the losses. The outcomes in analyzing such manifolds provide necessary data for modification. A number of trial and errors to minimize loss was carried out. Finally the best profile was adapted and was used for the stress analysis to minimize the stress below the allowable limit at the junction by adding reinforcement at junctions.

CFD is used for the analysis of the velocity and pressure distribution at different section of manifolds. The pressure and velocity distribution will then be used as a criteria modifying profile of manifold body.

Flotran CFD features of the Ansys v11 is used for the mathematical modeling of the flow through the profile. AutoCad 2006, Ansys Workbench 11 features is used for the mesh generation of the water volume inside the manifolds. The volume is imported in Ansys Flotran CFD for the analysis.

The jet velocity was expected as an output from the analysis

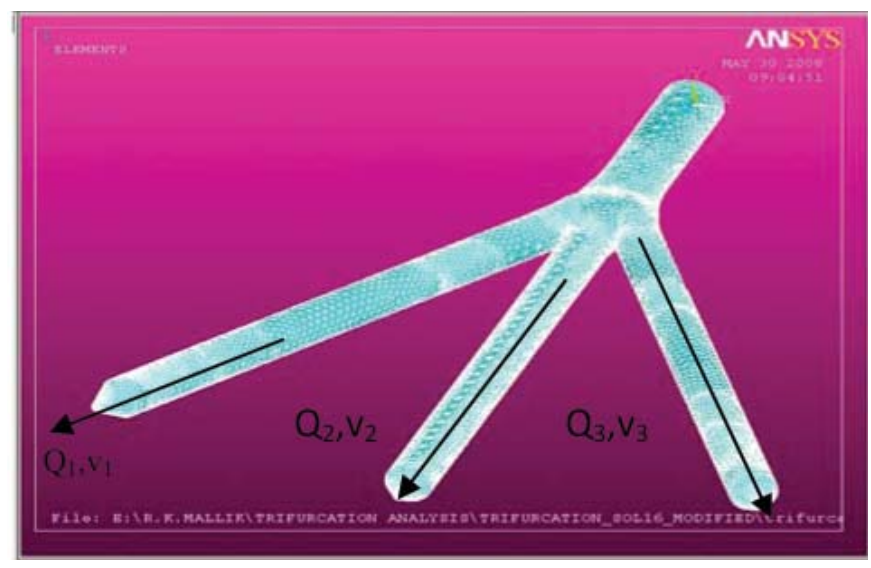

Fig.1. Ansys meshing of the trifurcation fluid control volume
If the net head of water and discharge at inlet is $\mathbf{h}$ and $\mathbf{Q}$ respectively then from the continuity equation for the equal discharge among three branching pipe,

Expected Discharge through each nozzle $=\mathrm{Q} / 3$

For no loss in the manifold system the velocity at each outlet nozzle $=\sqrt{2 g h}$

Corresponding area of the nozzle, $\mathrm{A}=Q / \sqrt{2 g h}$

Corresponding dia. of each nozzle $=\sqrt{4 A / \pi}$

This is the dia. of nozzle for no loss condition.

But due to losses in junction the neither all three velocity neither equal nor it is $\sqrt{2 g h}$

The evaluation of the real velocity at the outlet of the jet can help us to evaluate the loss by using the energy equation

Inlet Energy = Outlet energy + Loss in energy

Loss in energy = Outlet energy - Inlet energy

Inlet energy per unit time $=$ Work done by pressure per unit time + kinetic energy $=P Q+\frac{\rho Q v^{2}}{2}$

Outlet energy per unit time

$$
=\frac{\rho Q_{1} v_{1}^{2}}{2}+\frac{\rho Q_{2} v_{2}^{2}}{2}+\frac{\rho Q_{3} v_{3}^{2}}{2}
$$

Where $\rho$ : density of water

$$
\begin{aligned}
& Q \text { : Discharge in branching upstream } \\
& Q_{1}, Q_{2}, Q_{3} \text { : Discharge in Branching } \\
& v_{1}, v_{2}, v_{3}: \text { Velocities in branching }
\end{aligned}
$$

$Q, \rho, P$ are known input parameters, while $Q_{1}, Q_{2}, Q_{3}$ and $v_{1}, v_{2}, v_{3}$ can be determined from the Ansys Flotran CFD output by surface integral of velocity distribution of cross section near jet.

\section{ANSYS Flotran CFD}

Types of FLOTRAN Analyses

We can perform these types of FLOTRAN analyses:

- Laminar or turbulent

- Thermal or adiabatic

- $\quad$ Free surface

- Compressible or incompressible

- Newtonian or Non-Newtonian

- Multiple species transport

These types of analyses are not mutually exclusive. For example, a laminar analysis can be thermal or adiabatic. A turbulent analysis can be compressible or incompressible.

For the first trial, we use either of the Laminar Flow model or Turbulent Flow model. If differences in pressure and velocity field at a section appear significant after the first trial, we have to choose turbulence flow model for the next trial. Laminar and turbulent flows are considered to be incompressible since density is constant i.e. the fluid expends little energy in compressing the flow. 


\section{Flotran CFD analysis}

\section{Determining the Problem Domain}

The analysis of the losses in penstock pipe line up to trifurcation upstream was done to evaluate pressure and velocity as an inlet boundary condition for trifurcation. Boundary condition at out let of trifurcation is rather difficult process. It is preferable to model up to nozzle where there is free jet condition. Such modeling requires excessive computer memory almost difficult to solve with normal PC. If we focus on the losses due to trifurcation only then logically we can locate free jet zone some how near to the junction provided that it will not affect the junction velocity and pressure distribution.

\section{Determining the Flow Regime}

We need to estimate the character of the flow. The character is a function of the fluid properties, geometry, and the approximate magnitude of the velocity field. The Reynolds number can be used to decide weather the flow will be laminar or turbulent. Similarly, Mach no criteria can be used to evaluate the compressible and incompressible flow.

Reynolds number $=$ inertial force/viscous force, it should be greater than 2000 for turbulent flow. Mach no = Velocity of fluid/velocity of sound in fluid, which should be greater than 0.7 to activate the compressible flow model.

\section{Creating the Finite Element Mesh}

For the most accurate results, we should use mapped meshing. It more effectively maintains a consistent mesh pattern along the boundary. In some cases, we can use hexahedral elements to capture detail in high-gradient regions and tetrahedral elements in less critical regions. For flow analysis, especially turbulent, we should not use pyramid elements in the region near the walls because it may lead to inaccuracies in the solution.

In our case free meshing with tetrahedral element was used for simplicity in meshing it leads some degree of mass imbalance. The mass imbalance can be controlled by further fine meshing.

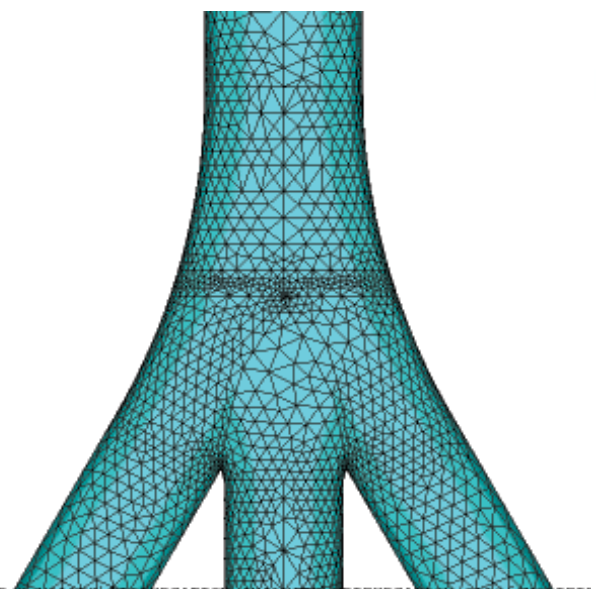

Fig.2, Tetrahedral meshing of fluid control volume near junction

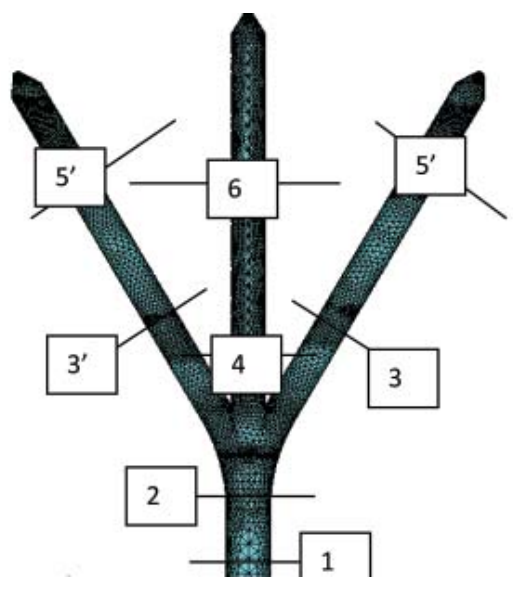

Fig.4.Different section of interest for recording output data

\section{Applying Boundary Conditions}

The free body diagram of the manifolds was considered for the analysis.

- The pipe wall was replaced by the zero velocity constrained.

- The net pressure near inlet surface of the manifold was directly applied (177m water head).

- The trial velocity at inlet is applied directly as $3 \mathrm{~m} / \mathrm{sec}$, $4 \mathrm{~m} / \mathrm{sec} . . . . .$. the velocity to generate input discharge corresponding to plant capacity.

The nozzle of the different trial dia. was attached with the trifurcation outlet. This helps us to apply pressure boundary condition at nozzle outlet as zero equivalents to atmospheric pressure.

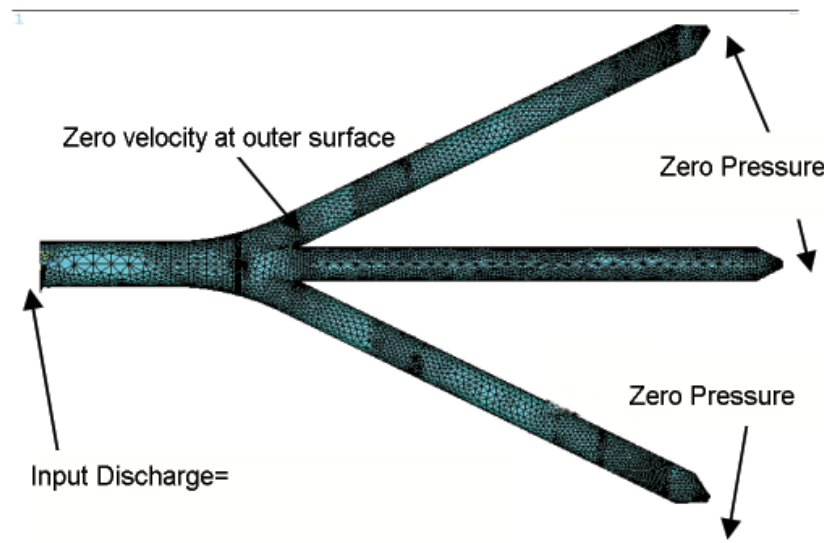

Fig.3. Boundary condition for most efficient trifurcation control volume

\section{Setting FLOTRAN Analysis Parameters}

This step includes selection of the flow models as laminar incompressible, laminar compressible, turbulent incompressible, turbulent compressible etc. The number of iterations, convergence option, and fluid properties should also be assigned in this step.

\section{Solving the Problem}

This step includes the solving process. We can monitor solution convergence and stability of the analysis by observing the rate of change of the solution and the behavior of relevant dependent variables. These variables include velocity, pressure, temperature, and (if necessary) turbulence quantities such as kinetic energy (degree of freedom ENKE), kinetic energy dissipation rate (ENDS), and effective viscosity (EVIS).

\section{Examining the Results}

The results are in the form of velocity distribution and pressure distribution and turbulence quantities distributions. The numerical integration is used to convert velocity distribution to average 
velocity at outlets. The average velocity at inlet and outlets are then used to evaluate the loss in manifold.

The loss in energy is made as small as possible by adjusting the geometrical profile of manifold at joint.

This is the trial and error process. The modification is made till the loss in the manifold is equivalent to $0.42 \%$.

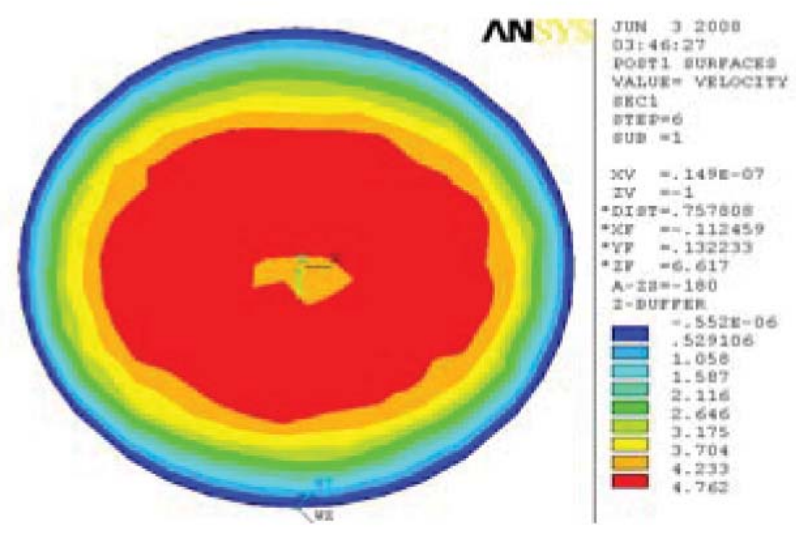

Fig.5.Velocity distribution at section 1-1

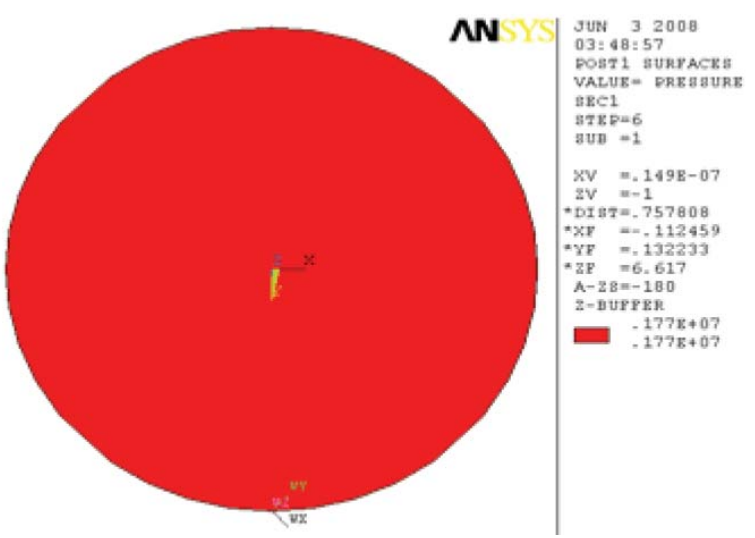

Fig.6.Pressure Distribution at section 1-1 Uniform pressure of $177 \mathrm{~m}$ water head

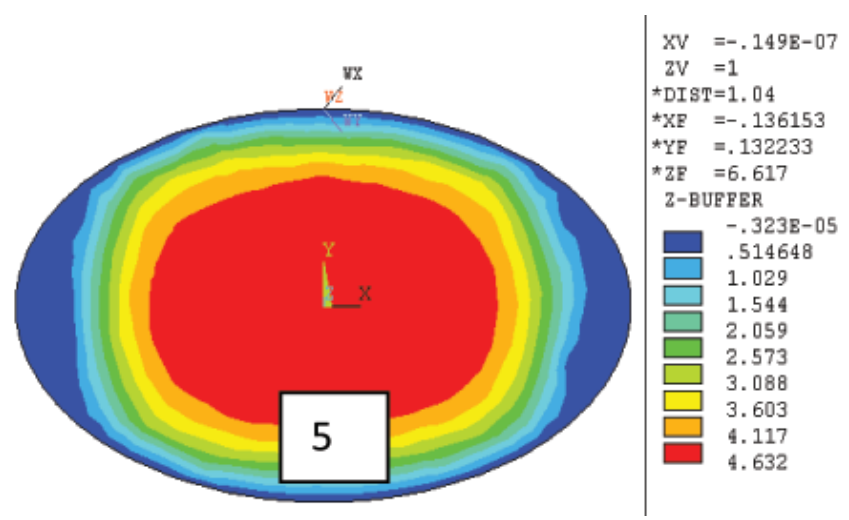

Fig.7.Velocity distribution between section 1 and 2

The velocity distribution profile for section at junction indicates the causes of loss of energy near junction. These velocity profiles are for the most possible efficient geometry of the trifurcation junction.

\section{Conclusions from most efficient profile}

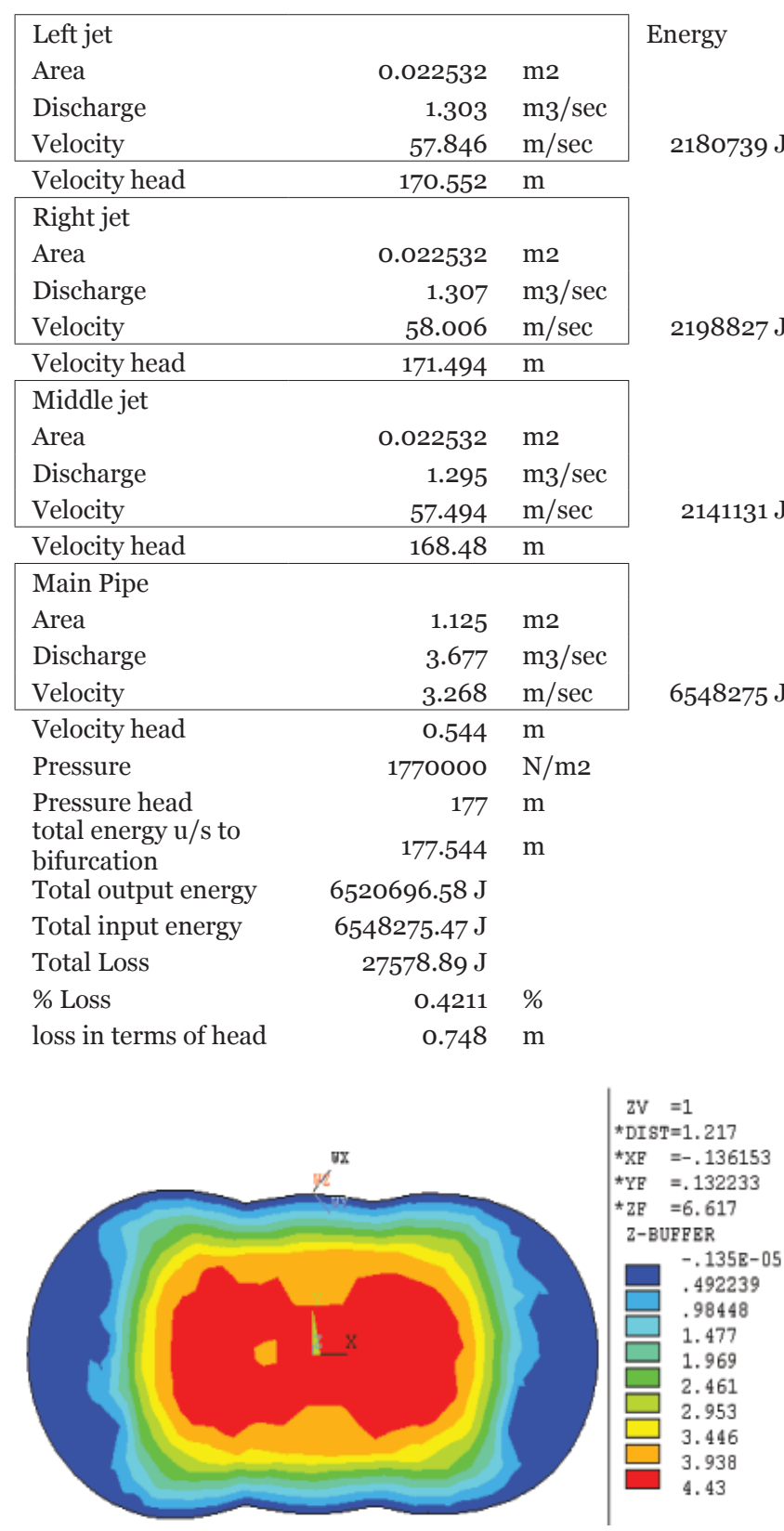

Fig.8.Velocity distribution between section 1 and 2

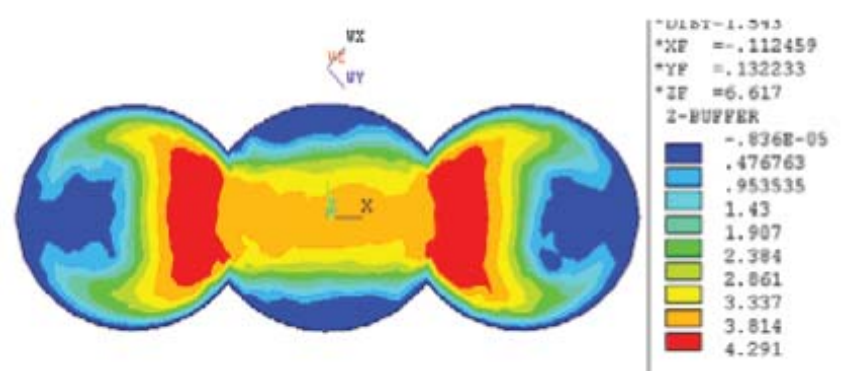

Fig.9.Velocity distribution in section $2-2$

\section{Stress Analysis at J unction}

According to the ASME Code, the non-embedded penstock pipe may be designed under the following condition. 

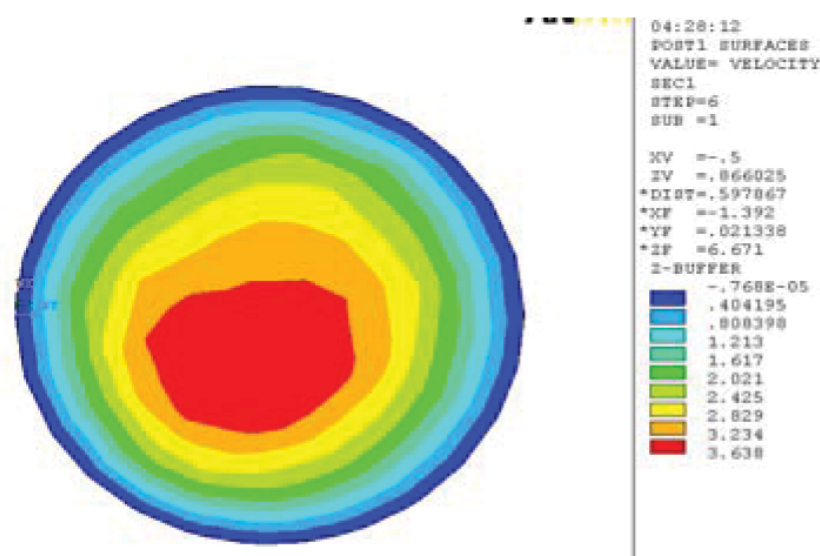

Fig.10.Velocity distribution in sections 5-5

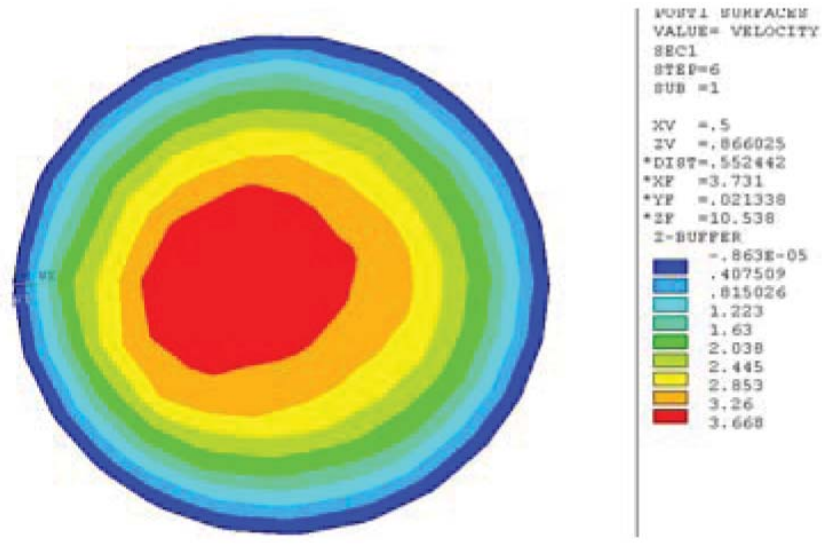

Fig.11.Velocity distribution in sections 5'-5'

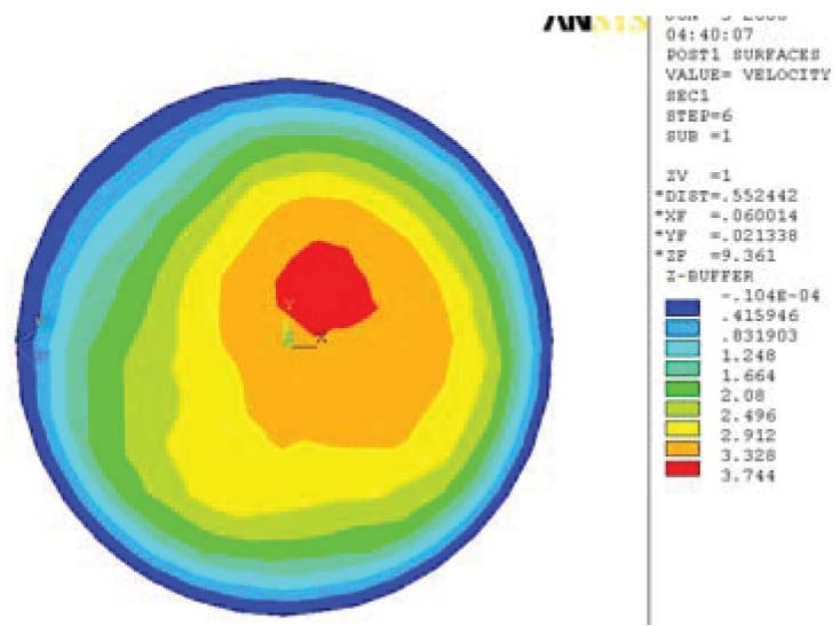

Fig.12.Velocity distribution in sections 6-6

Normal condition:

This condition gives the allowable stress $=138.67 \mathrm{Mpa}$

\section{Intermittent condition:}

This condition gives the allowable stress $=184.89 \mathrm{Mpa}$

Emergency condition:

This condition gives the allowable stress $=\mathbf{2 5 0} \mathrm{Mpa}$

\section{Exceptional conditions:}

It includes malfunctioning of control equipment in most adverse manner and shall not be used as the basis of design.

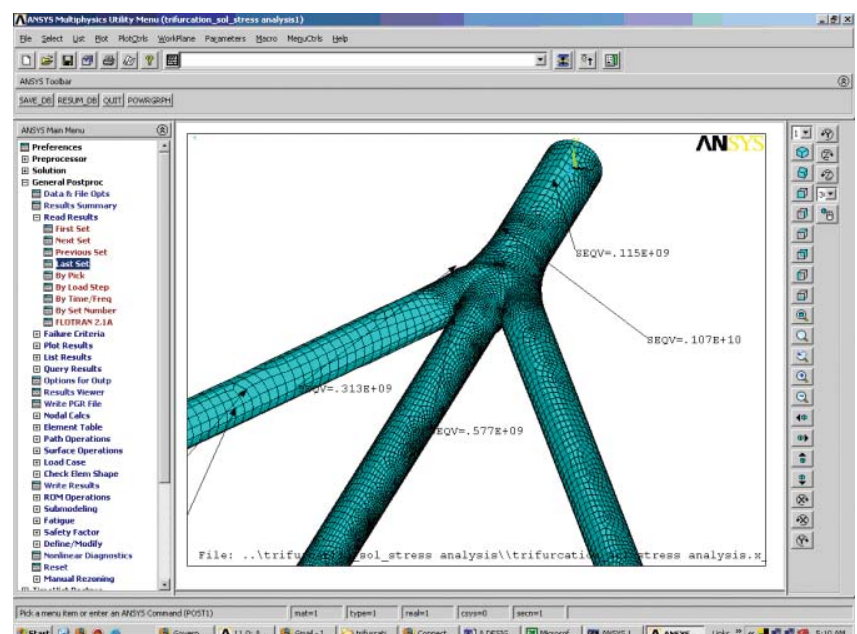

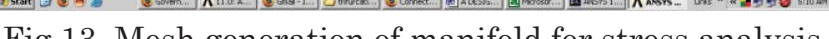

If the maximum stress does not exceed the specified minimum ultimate tensile strength, the structural integrity of the penstock is reasonably assured.

Precautions must be taken to minimize the probability of occurrence and effects of the exceptional condition.

In this analysis the stress was supposed not to be increase above $138 \mathrm{Mpa}$ in normal water pressure + water hammer pressure. For the combination of normal flow condition and the earthquake effects the whole penstock unit should be studied.

The Von-misses stress criterion was used for checking the yield at the zone of stress concentration

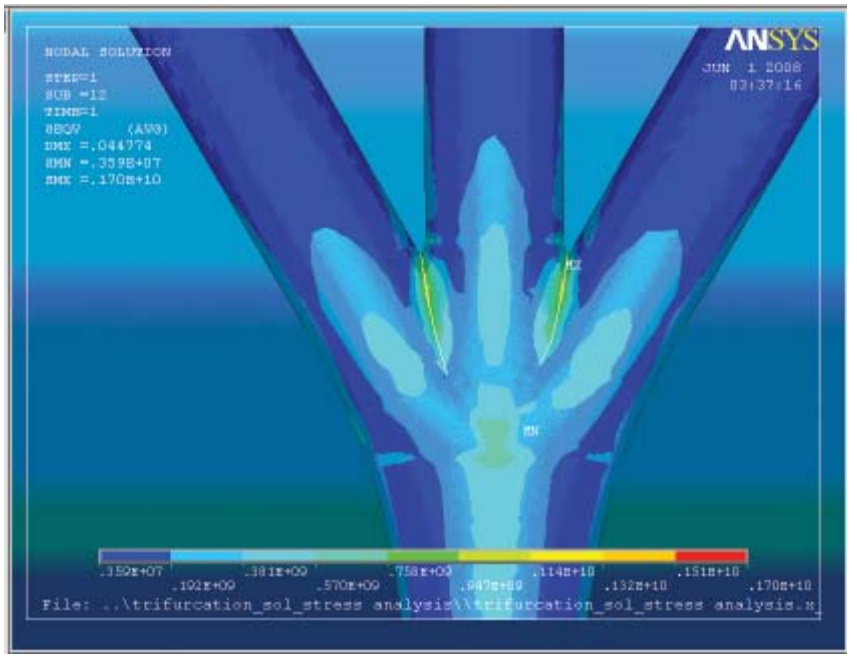

Fig.14. Von-misses stress distribution showing stress concentration near junction.

\section{Examining the Results}

The stress at the junction of the manifold was observed exceeding at normal running condition. The problem was resolved by adding extra reinforcing plate at junction in the form of $\mathrm{T}$ section

\section{Conclusions}

Around 20 models were checked for the loss of the head due to geometry of manifold between common profiles 


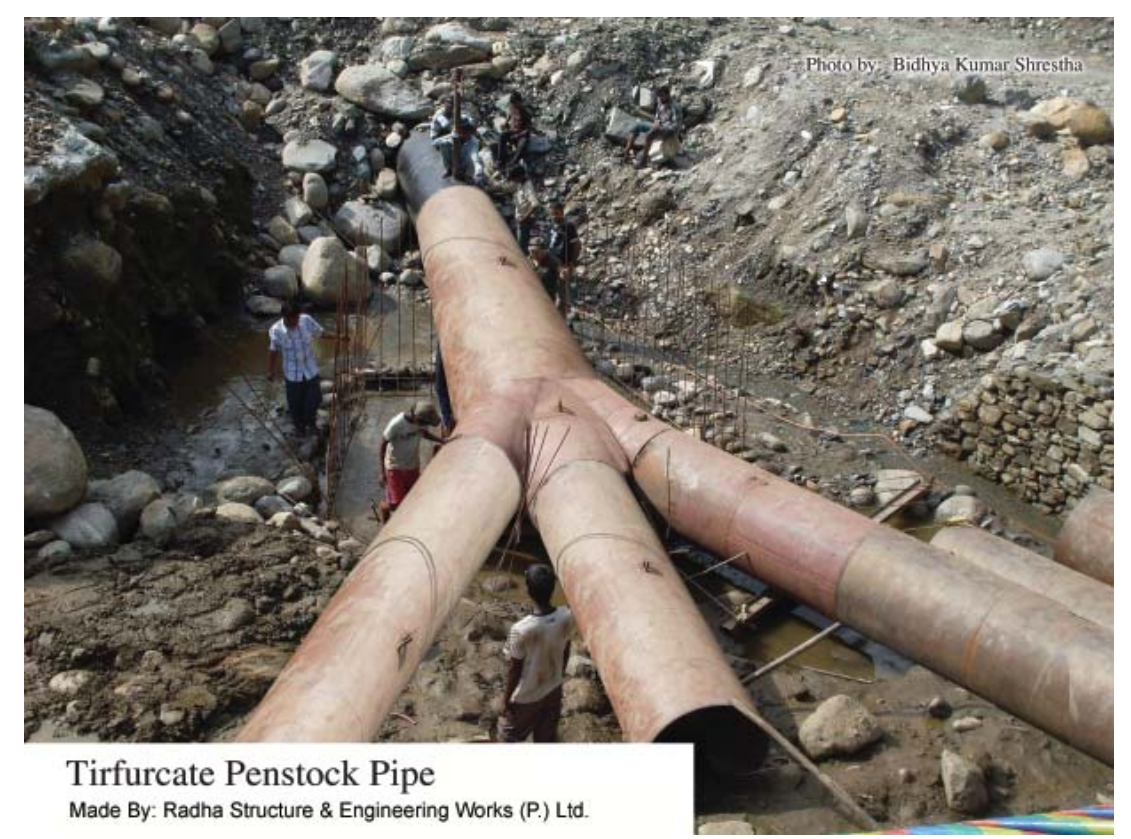

to most efficient profile. Only result for the most efficient profile is presented in this paper.

The Study indicates that the profile can be better tuned with the finite element application for fluid flow (CFD).

The flow distribution in case of common profile is rather disturbed to longer zone of the trifurcation system. In case of most efficient profile the flow distribution is disturbed slightly to smaller length of the trifurcation system.

The second profile was taken for the fabrication to limit loss in head less than one meter head of water

R. K. Mallik completed his M.Sc. in Structural Engineering from IOE in 2005. He has worked for Radha Structure Engineering \&Works and many consultancy as a Structural Engineer. These days he is involved as research engineer at NSET. He has started the finite element modeling for the analysis of the complex profile of hydropower for the first time in Nepal such as Bifurcation modeling for Heldung Hydropower and Lower Piluwa Hydropower.

Besides other different researches in structural field, he was the developer of the analysis and design methodology for the 3-Dimensional flow modeling in the trifurcation for Madi Khola small hydropower for the first time in Nepal.

Corresponding address: rkmallik@gmail.com

Paras Paudel completed B.E. in Mechanical Engineering in 2006. He was involved in various research activities in Robotics club of IOE. Heis currently working as a Mechanical Engineer at Nepal Airlines Corporation.

He was involved in the 3-Dimensional flow modeling and surface developments of trifurcation for Madi Khola small hydropower.

Corresponding address: paras.paudel@gmail. com

\section{References}

ANSYS Fluid Analysis Guide, ANSYS Release 10.0.002184, ANSYS,Inc. Southpointe 275 Technology Drive Canonsburg, PA 15317, August 2005.

Chen Y.S., Kim S.W., 1987, Computation of Turbulent Flows Using an Extended k- $\varepsilon$, Turbulence Closure Model, NASA CR-179204

Buntić Ogor I., Dietze S., Ruprecht A., Numerical Simulation of Flow in Turbine -99 Draft Tube, Proceedings of the third IAHR/ ERCOFTAC workshop on draft tube flow 8-9 December 2005, Porjus, Sweden

T.J. Chung Computational Fluid Dynamics, University of Alabama in Huntsville, Cambridge University Press, First published at 2002

Hydropower in Norway, Mechanical Equipment, A survey prepared by Arne Kjølle, Professor Emeritus Norwegian University of Science and Technology. December 2001

Xiaodong Wang, On Mixed Finite Element Formulations for Fluid-Structure Interactions, $\mathrm{PhD}$ Thesis in Applied Mechanics, MIT, J une 1995

F. Nobile: Numerical Approximations of Fluid-Structure Interactions with Applications to Haemodynamics, Ph. D. Thesis, EPFL Lausanne (CH), 2001

Ellen Kock, Lorraine Olson, Fluid-Structure Interaction Analysis By The Finite Element Method-A Variational Approach, International Journal for Numerical Methods in Engineering,Volume-31,Issue-3, 24 June 2005

University of Alberta ANSYSTutorials- www.mece.ualberta. $\mathrm{ca} /$ tutorials/ ansys

P. Colella and E.G. Puckett, Modern Numerical Methods for Fluid flow, UC Berkeley E266A course notes

By Thomas H. Pullia, Solution Methods in Computational Fluid Dynamics, Based on notes from a VKI lecture series in 1986. 